\title{
Appareil
}

12 | 2013

Walter Benjamin. Politiques de l'image

\section{De la compénétration des espaces chez W. Benjamin aux Unités d'habitation de Le Corbusier}

Jean-Louis Déotte

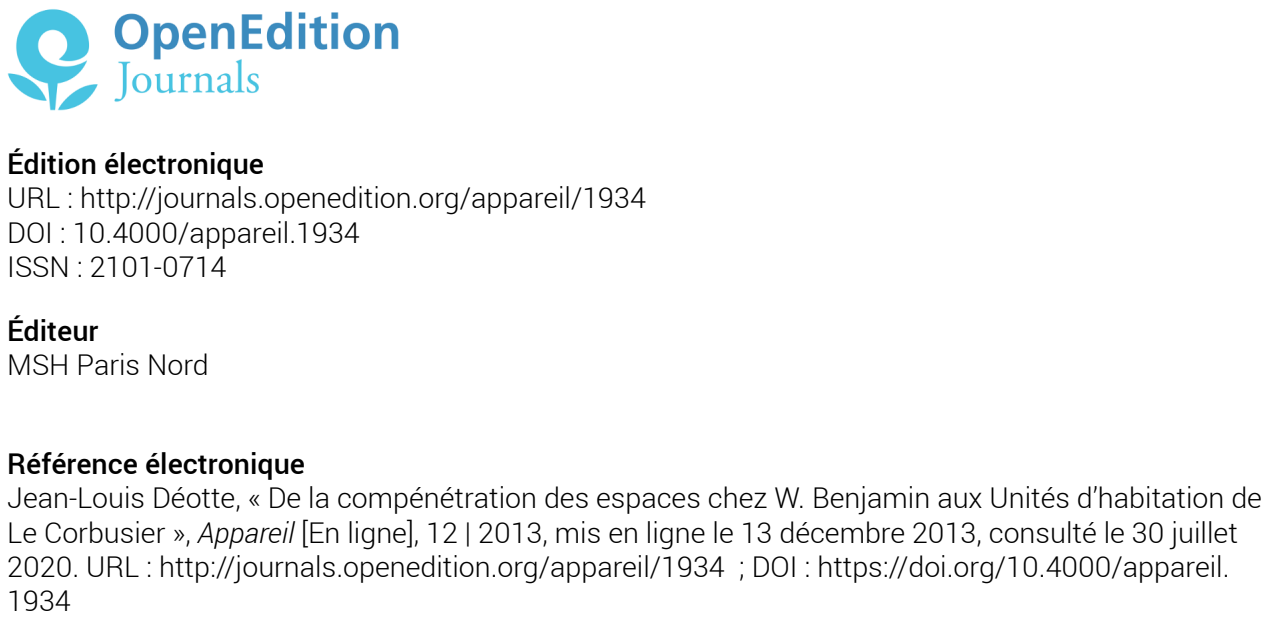

Ce document a été généré automatiquement le 30 juillet 2020.

\section{(c) (1) ()}

Appareil est mis à disposition selon les termes de la Licence Creative Commons Attribution - Pas d'Utilisation Commerciale - Pas de Modification 4.0 International. 


\title{
De la compénétration des espaces chez W. Benjamin aux Unités d'habitation de Le Corbusier
}

\author{
Jean-Louis Déotte
}

1 On posera par hypothèse que Walter Benjamin aborde la question de la politique par deux grands thèmes :

2 1. La fantasmagorie originaire qui donne une consistance de rêverie collective à la spatialité commune, comme si la sensibilité commune des sociétés humaines, dès la Préhistoire (Urgeschichte, avec le double sens d'époque et d'originarité), avait eu une consistance de rêverie, comme si l'espace avait toujours la consistance de l'image ${ }^{1}$.

W. Benjamin aura accompli un déplacement très important par rapport au marxisme : ce ne sont plus les énoncés qui entretiennent l'illusion (l'aliénation par l'idéologie), mais les images. On passe ainsi d'une conception de l'aliénation en termes d'idéologie à une conception du sol commun en termes de fantasmagorie. Ce qui propulse au premier plan le monde fantastique et magique des images, toujours perçues à travers des dispositifs et appareils optiques. D'où le terme de fantasmagorie, très en vogue au $\mathrm{XIX}^{\mathrm{e}}$ siècle pour caractériser la littérature d'Hoffmann ou de Poe en particulier. Quant aux énoncés, ils acquièrent le statut d'archives véridiques, de purs documents (comme plus tard chez Foucault) qui n'ont pas besoin d'être interprétés (il suffit de les exposer dans leur littéralité : ce qui sera fait dans Paris et revendiqué par lui-même comme son mode d'écriture). W. Benjamin rompt avec l'herméneutique quand il proclame qu'il expose des textes comme des objets et qu'il ne les interprète pas. Paris doit donc être considéré comme l'exposition d'une collection d'objets non fantasmagoriques.

4 2. L'espace politique, à la différence de chez Arendt-Nancy, n'est pas un simple espace d'entre exposition des singularités les unes aux autres (Nancy: l'espace de comparution) opposé à un lieu privé et intime (la maisonnée chez Arendt). Car chez ces auteurs, l'espace politique est indifférencié et non déterminé sur le plan urbanistique et architectural, même s'il y a chez eux une approche de la ville ${ }^{2}$. Au contraire, il faut penser l'espace psycho-sociologique à partir de l'opposition structurante habitation/ 
communication. Par exemple, au XIX $x^{e}$ siècle : l'appartement entendu comme étui pour un particulier qui y laisse ses traces versus le passage urbain qui englobe la foule des anonymes.

Jusqu'ici l'œuvre de W. Benjamin a été analysée à partir de la temporalité, en particulier dans mon ouvrage L'Homme de verre: esthétiques benjaminiennes ${ }^{3}$. Il en allait de même chez F. Proust : L'Histoire à contretemps : le temps historique chez Walter Benjamin ${ }^{4}$. Etc.

À partir de mon Walter Benjamin et la forme plastique ${ }^{5}$, en insistant sur la découverte de l'architecture pré-moderne française, effectuée à partir de sa lecture éblouissante pour lui de Giedion : Construire en France, construire en fer, construire en béton $(1928)^{6}$, on peut montrer que $\mathrm{W}$. Benjamin va développer une problématique de la spatialité, en termes de topologie. Ce qui implique qu'entre la forme et le contenu d'une chose, il y a à la fois déformation et conservation d'invariants, géométriques par exemple ${ }^{7}$.

7 On peut donner une image d'un espace topologique : une membrane de caoutchouc. Ainsi une tasse peut être dite homéomorphe d'un tore (solide géométrique présentant un tube courbé refermé sur lui-même).

Et réciproquement. Cette géométrie a été élaborée à partir des travaux de Poincaré et a connu depuis une grande complexification.

Elle est aujourd'hui à la base des architectures "non-standard» élaborées par ordinateur. Je renvoie aux travaux de G. Teyssot sur la numérisation de l'architecture ${ }^{8}$. La ligne droite est alors détrônée, par l'invention de nouvelles courbes mathématiques et de nouveaux logiciels. Les volumes privilégient des formes bulbaires. On peut se demander néanmoins si toute architecture projective, et même tout l'art occidental projectif des Florentins $\mathrm{du} \mathrm{xv}^{\mathrm{e}}$ siècle jusqu'aux collages dadaïstes, ne sont pas topologiques, mais d'une manière limitée car soumis aux critères de la représentation.

Giedion avait développé dans son ouvrage-manifeste deux grands thèmes :

1. L'architecture pré-moderne était contradictoire, industrielle d'un côté, artistique de l'autre. Technique versus rêveuse. Fonctionnaliste versus « de mauvais goût », passéiste. Il reviendra aux Modernes de dépasser cette contradiction (Perret, Sauvage, Garnier, Loos, Le Corbusier) en brisant le carcan fantasmagorique, ce qui correspondrait selon Benjamin à une sortie hors du rêve dans lequel le XIX siècle s'était enfermé, par une sorte de réveil (Erwachen) révolutionnaire.

13 L'habitation (das Wohnen) devient un enjeu politique central, on en veut pour preuves les Unités d'habitation de Le Corbusier ("Cités radieuses») qui devinrent l'objet d'âpres polémiques, voire de tentatives de destruction. L'idéal réactionnaire de la droite, c'est le pavillon de banlieue, le lotissement péri-urbain, la propriété privée du chez-soi, le fanatisme nazi du toit gothique. Conséquence aujourd'hui : les immenses lotissements qui dévorent les terres agricoles, conduisant à une impasse écologique et économique (le prolongement de tous les réseaux de distribution de l'énergie, de l'eau, etc., le bétonnage des sols). Il faut donc revenir à la question de l'habitat collectif comme question politique essentielle.

14 2. Dans sa lutte contre la maison devenue monument, Giedion mettait l'accent sur l'entrecroisement (Verschränkung) des espaces. D'où sa condamnation des villas modernistes de Mallet-Stevens. Dès lors, son histoire de l'architecture est téléologique : 
il y a un accomplissement qui a pour nom Le Corbusier, dont il ne connaissait alors que quelques réalisations (surtout les villas).

W. Benjamin va lancer à Giedion un défi paradoxal: là où celui-ci avait pensé les rapports infrastructure/superstructure (ossature technique / façades néo-classiques), lui (W.B.) va déplacer le problème. Ce qui en apparence l'éloigne radicalement du champ de l'architecture. Soit, propose-t-il arbitrairement, penser le rapport : processus physiologique/rêves. De l'architecture on passe au corps du collectif :

La collectivité aussi est de nature corporelle. Et la phusis qui pour elle s'organise en technique ne peut être produite dans toute sa réalité politique et matérielle qu'au sein d'un espace d'images avec lequel l'illumination profane nous familiarise. Lorsque le corps et l'espace d'images s'interpénètreront en elle si profondément que toute tension révolutionnaire se transformera en innervation du corps collectif, toute innervation corporelle de la collectivité en décharge révolutionnaire, alors seulement la réalité sera parvenue à cet autodépassement qu'appelle le Manifeste communiste ${ }^{9}$.

Pour comprendre la portée de ce déplacement, du «matérialisme métaphysique » au "matérialisme anthropologique», il faut revenir sur un certain nombre de remaniements benjaminiens :

W. Benjamin concevait l'imagination, en particulier après ses expériences hallucinogènes marseillaises, comme une "faculté » essentielle de déformation et non de destruction. Ce qui l'amenait à concevoir que la création artistique consiste en une déformation de formes culturelles héritées ${ }^{10}$. On peut faire l'hypothèse que ce type d'expérience, où l'opposition sujet/objet est déconstruite, est pour lui la matrice de toute expérience possible, quelque chose comme l'écran du rêve pour la psychanalyse, ou la forme du rideau ondulant dans le vent servant de support aux images, aux objets et à leur perception subjective, décrite dans les protocoles d'expérience à Marseille ${ }^{11}$. On peut parler à la suite de J. Selz d'une "rideaulogie " (travaux de S. Amy) pour donner un exemple de ce que W. Benjamin appelait « surface absolue » (Fragments).

L'expérience hallucinogène est probablement l'expérience la plus authentique et la plus originaire dont il montre l'absence et donc la nécessité dans une perspective comme celle de la philosophie kantienne (Pour une philosophie qui vient...), philosophie jugée trop positiviste, car réduite à l'objectivation du monde.

On abordera deux aspects : la relation forme/contenu, la relation couleur/surface. de la chaussette est une boule, une bourse, son contenu, c'est la chaussette dépliée ${ }^{12}$. Dans le passage d'un stade à l'autre, il y a bien conservation d'une entité : la chaussette, malgré une déformation radicale. Le contenu est donc la forme déployée selon le principe de l'évocation, qui n'est ni une symbolisation, ni une métaphorisation au sens classique, mais une allégorisation ou une ruinification sans négativité.

21 Il y a donc rupture avec les conceptions platoniciennes, aristotéliciennes, voire simondoniennes de la relation forme/matière. Il faudrait ici reprendre la critique de l'hylémorphisme aristotélicien par Simondon en posant la question: quel est le principe de l'individuation de la chose chez W. Benjamin?

On peut répondre à cela par sa conception de la couleur. La couleur n'est pas comme le dessin qui a besoin d'un support à partir duquel il se distingue. La couleur se répand comme une tache d'encre sur le papier buvard : elle génère sa forme au fur et à mesure qu'elle se répand. Dès lors, comme réponse à la question de l'individuation de la forme/ 
matière, la réponse est: le nom propre. C'est le nom qui de l'extérieur individue la forme, sur le modèle de la création divine (Sur le langage en général...). Pour Dieu, créer et nommer sont la même action.

W. Benjamin renouvelle donc de fond en comble la problématique du «matériau artistique ». Qu'en sera-t-il chez Adorno?

La peinture privilégiée par W. Benjamin sera donc liquide comme chez l'artiste Germain Roesz: aquarelle, décalcomanie, irisations de la bulle de savon, etc. À l'exemple des couleurs diaphanes de l'arc en ciel. Importance de l'art Chinois, du retable d'Issenheim et des maisons de verre de Scheerbart.

Ce que découvre W. Benjamin à la lecture de Giedion, c'est que l'architecture prémoderne était incohérente, puisque si l'infrastructure industrielle était en métal, la superstructure, les façades étaient néo-classiques. La forme architecturale moderne devra donc être le produit d'un montage puisque c'est le principe de la production industrielle des éléments, et non plus une forme "harmonieuse ». Il en ira de même pour la production littéraire, exposant des éléments qui devront parler d'eux-mêmes : littéralité de l'exposition des archives qui devait être le principe d'exposition de Paris, capitale du XIX siècle.

On parlera plus précisément de forme plastique quand le principe de technicité sera la clef des rapports forme/contenu. Soit une adéquation de la forme et du contenu selon le principe de la résolution d'un problème technique, d'une innervation de la nature (le corps collectif) par la technique. Ce qui bouleverse la notion d'intérieur : par exemple sa conception fonctionnaliste du moteur de l'automobile, lequel est entendu comme intérieur (opposé à la prétendue intériorité psychologique du particulier). La forme plastique technique devient à l'époque de la technique ce qui s'impose à tous les arts, à la littérature en premier lieu. Le Paysan de Paris d'Aragon, texte de montage, est plus essentiel que Madame Bovary, chère à l'esthétique de Rancière.

Appliquées à la relation espace privé / espace collectif, différentes époques de la compénétration seront considérées par W. Benjamin.

\section{La Renaissance avec le théâtre Olympique de Palladio/Scamozzi à Vicenza ${ }^{13}$}


Figure 1

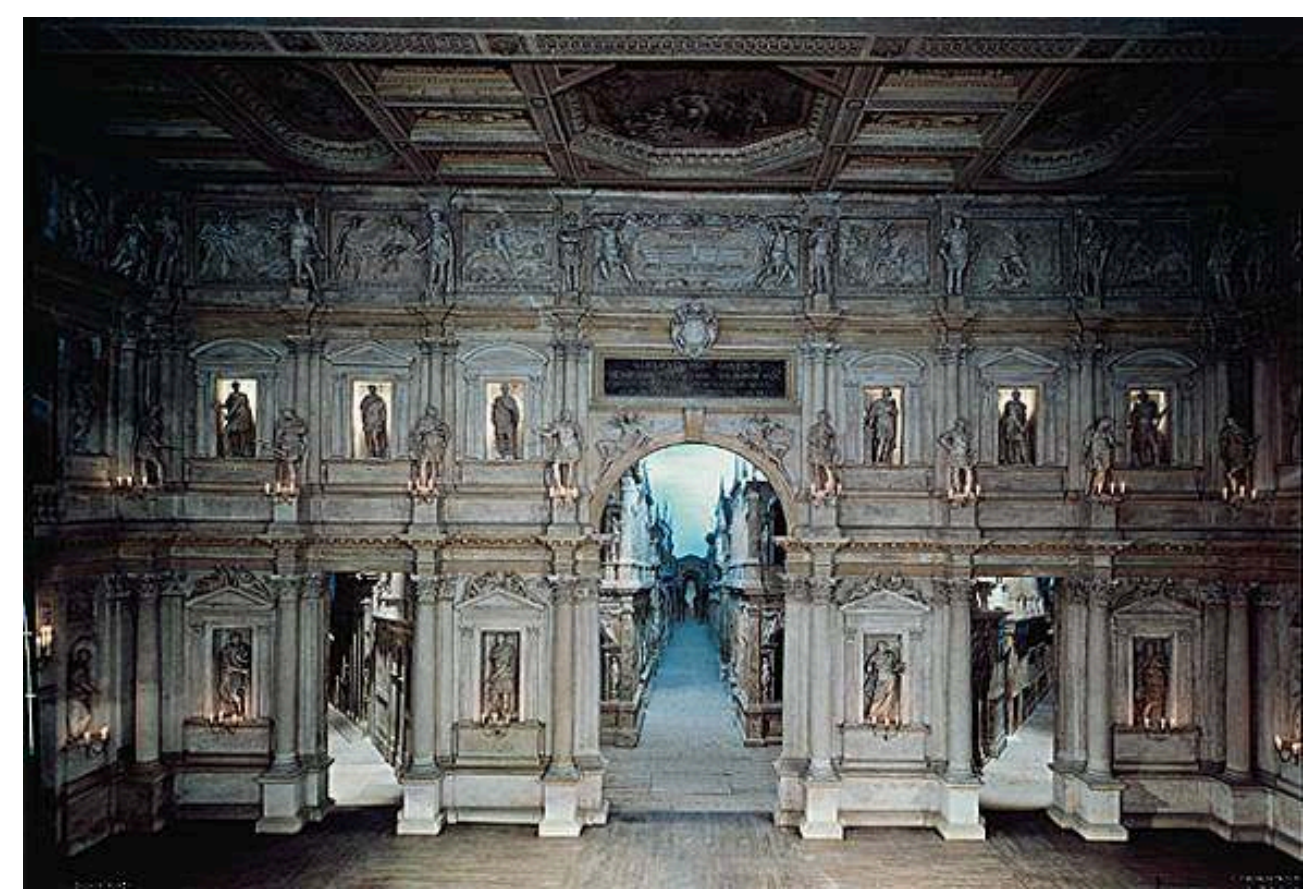

Vicenza : scène du théâtre Olympique maison et de l'espace collectif de la rue à l'aune de la représentation perspectiviste. S'il y a un échange des deux types d'espace, c'est sous l'égide de la représentation. En apparence la compénétration est parfaite, mais il s'agit d'une fantasmagorie objectiviste comme le confirmeront les textes sur les perspectives haussmanniennes (Paris, capitale du XIX siècle).

Les passages entraînent une dissociation de la foule et du particulier réduit à ne concevoir l'univers qu'en fonction de son étui propre (appartement). Critique de l'appartement bourgeois dans Expérience et pauvreté : saturation des traces versus rêverie collective soumise à la distraction agissant sur les processus physiologiques du corps de la foule. Le passage appareille la ville, non plus en termes projectifs, mais immersifs. Dans un passage, la foule absorbe physiologiquement l'extériorité (le monde atmosphérique, l'architecture, les marchandises), c'est la raison pour laquelle elle deviendra la proie des partis totalitaires, que l'on peut considérer, comme le fera Arendt, par leur fluidité idéologique ${ }^{15}$. Il en va de même pour ses rapports immédiats au cinéma : une foule de spectateurs est d'entrée de jeu pré-totalitaire (esthétisation de la politique $)^{16}$. On pourrait parler de la liquidité de la modernité, dans un sens proche de Zygmunt Bauman, mais d'une manière littérale, car pour Benjamin, à la suite d'Aragon parcourant le passage de l'Opéra ${ }^{17}$, la vie dans un passage est celle d'un aquarium :

Quelle ne fut pas ma surprise, lorsque, attiré par une sorte de bruit machinal et monotone qui semblait s'exhaler de la devanture du marchand de cannes, je m'aperçus que celle-ci baignait dans une lumière verdâtre, en quelque manière sous-marine, dont la source restait invisible. Cela tenait de la phosphorescence des poissons, comme il m'a été donné de la constater, quand j'étais encore enfant sur la jetée de Port-Bail, dans le Cotentin, mais cependant je devais m'avouer que bien que des cannes après tout puissent avoir les propriétés éclairantes des habitants de la 
mer, il ne semblait pas qu'une explication physique pût rendre compte de cette clarté surnaturelle et surtout du bruit qui emplissait sourdement la voûte. Je reconnus ce dernier: c'était cette voix de coquillages qui n'a pas cessé de faire l'étonnement des poètes et des étoiles de cinéma. Toute la mer dans le passage de l'Opéra. Les cannes se balançaient doucement comme des varechs ${ }^{18} .$.

S'il y a une fantasmagorie propre au passage, ce n'est pas contrairement à ce qu'écrit Marc Berdet dans un livre récent ${ }^{19}$, le fétichisme de la marchandise, au sens de Marx, soit l'illusion d'un échange autonome des marchandises, ni les féeries coloniales du Crystal palace (Londres, 1851), mais les naïades pré-pubères des décors de l'univers de l'Art nouveau qui privilégient les lignes courbes du kitsch.

Figure 2

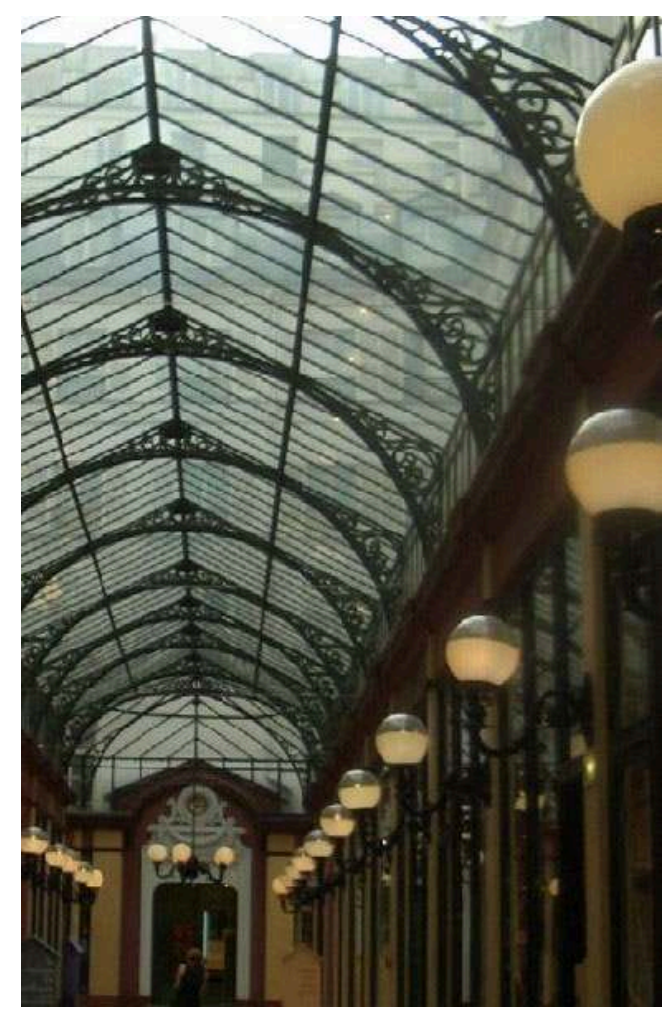

Galerie Colbert à Paris

L'individu devient un atome social : le particulier, qui n'a d'existence subjective qu'étant modelé par l'étui (l'appartement) dans lequel il vit. Entre la forme-étui et l'habitant, la relation est topologique. L'étui donne forme à son contenu comme le ferait un moule (W. Benjamin décrit le xix siècle comme multipliant les étuis pour tous les objets précieux). Inversement, l'habitant trouve dans l'étui le seul support disponible pour laisser les traces de son passage éphémère dans ce monde. L'appartement devient très vite totalement saturé de traces et d'enveloppes. Les analyses de W. Benjamin sont merveilleuses de précision : il ouvre la voie à ce qui deviendra avec Henri Lefebvre une sociologie critique de la vie quotidienne, qui sera reprise par les situationnistes. L'appartement et la capacité d'inscrire les traces de l'occupant vont devenir un enjeu architectural, urbanistique et finalement politique, ce que comprendront bien les «modernes », Bauhaus inclus. 


\section{Naples ${ }^{22}$}

Car pour W. Benjamin, si le XIX siècle a vraiment inventé l'appartement comme support des traces de la singularité, en cela il est préhistorique (Urgeschichte). Pour lui, le $\mathrm{XIX}^{\mathrm{e}}$ siècle a inventé la Préhistoire, non comme discipline scientifique, mais parce qu'il a réalisé un phénomène originaire (Goethe). Car la relation originaire entre une singularité et son enveloppe, son étui, c'est celle du fotus dans le ventre maternel (métaphore abondamment reprise par Sloterdijk).

Mais pour lui, si dans ce sens le $\mathrm{XIX}^{\mathrm{e}}$ siècle est "authentique ", " au plus près de l'origine ", c'est au nom d'une régression totale. L'archaïque, s'il est le prototype de la relation humaine, est parfaitement régressif. Car ce moule est totalement séparé du moule du collectif: le passage. Donc l'appartement-étui ne peut générer qu'un rêve individuel : celui de la possession du monde entier par des traces de ce dernier, les « souvenirs » touristiques des différents voyages.

Le $\mathrm{XIX}^{\mathrm{e}}$ siècle est donc bien celui de l'échec total de l'articulation psycho-sociologique, et donc de la compénétration : l'opposition privé/public se réalise dans la régression la plus radicale : rêve/processus physiologiques.

Il faudra donc attendre ces nouveaux barbares que furent Loos et Le Corbusier pour que l'appartement devienne " poreux, transparent, lumineux et ouvert sur l'extérieur ${ }^{20} »$. Il faudra donc sortir de la Préhistoire, entrer dans l'Histoire, ce qui suppose prendre en considération le passage entre appartement et espace de communication. C'est de ce point de vue qu'il faudra considérer les termes de "porosité » et de "transparence » qui ne peuvent convenir à la seule description de l'appartement.

\section{Moscou la révolutionnaire ${ }^{21}$}

La solution est-elle du côté de Moscou la révolutionnaire ? Or, l'appartement moscovite ne permet à l'individu aucune inscription de traces, du fait de la mobilisation totale de la masse par la politique au moment de la contre-révolution stalinienne (1927).

C'est à Naples que la compénétration est pleinement réussie. Car il y a une réversibilité de toutes les possibilités du rapport selon la détermination du principe de porosité. La compénétration est alors littérale (pierre de lave) et topologique-politique ${ }^{23}$.

La porosité de la pierre de lave napolitaine, voire du village autochtone sud-africain (peuple Hottentot), se réalisera dans la ville moderne grâce au béton, comme chez Le Corbusier : Cités radieuses de Marseille, Rezé, Briey en forêt, Firminy. L'artisanat auquel Le Corbusier a dû recourir dans l'urgence et la pauvreté des moyens l'ont empêché de lisser le béton qui a été livré - et conservé depuis lors - brut de décoffrage. Alors la matière est adéquate à la forme. Il y a eu chez Le Corbusier une décision : accepter ce que lui livrait les bâtisseurs en ne reniant pas cette pauvreté de l'expérience, d'où ce «brutalisme » dans la fabrication des pilotis par exemple ${ }^{24}$.

Finalement, l'Unité d'habitation articule d'une manière cohérente espaces privés des appartements et espaces collectifs (appartements encastrés, services communs innovants, rues intérieures, toit paquebot, crèche, école, commerces, salles de réunion, théâtre, etc.). Benjamin aura gagné son pari contre Giedion : il sera plus corbuséen que lui. Et surtout plus politique, surtout si on le compare aux réalisations d'un autre disciple de Le Corbusier comme Niemeyer (Brasilia!). Le Corbusier a pu réaliser des architectures qui étaient des chefs d'œuvre de sculpture (Ronchamp), mais il a aussi su réaliser de véritables cités qui sont des microcosmes de la ville, c'est-à-dire des 
laboratoires politiques que leurs actuels habitants ne déserteraient pour rien au monde.

Figure 3

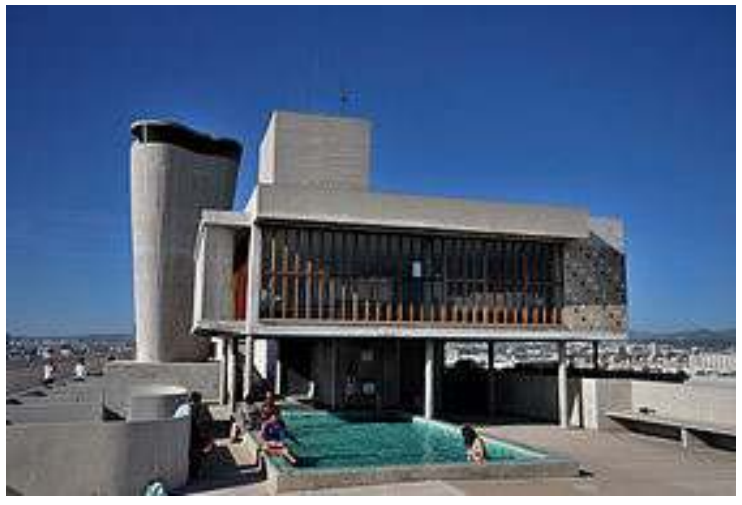

Marseille la terrasse de la cité radieuse

(c) Licence Creative Commons «Paternité - Partage des conditions initiales à l'identique » http:// creativecommons.org/licenses/by-sa/3.0/deed.fr

Figure 4

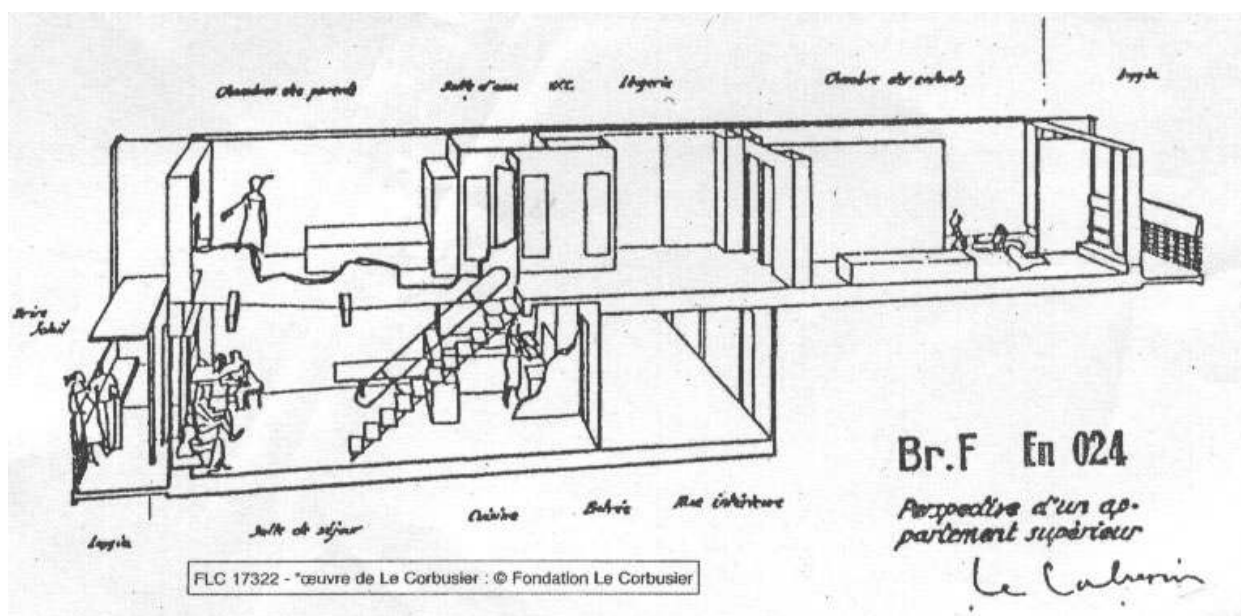

Le Corbusier : Cité radieuse de Marseille, coupe des rues intérieures, toit collectif, pilotis de la cité radieuse de Marseille. 
Figure 5

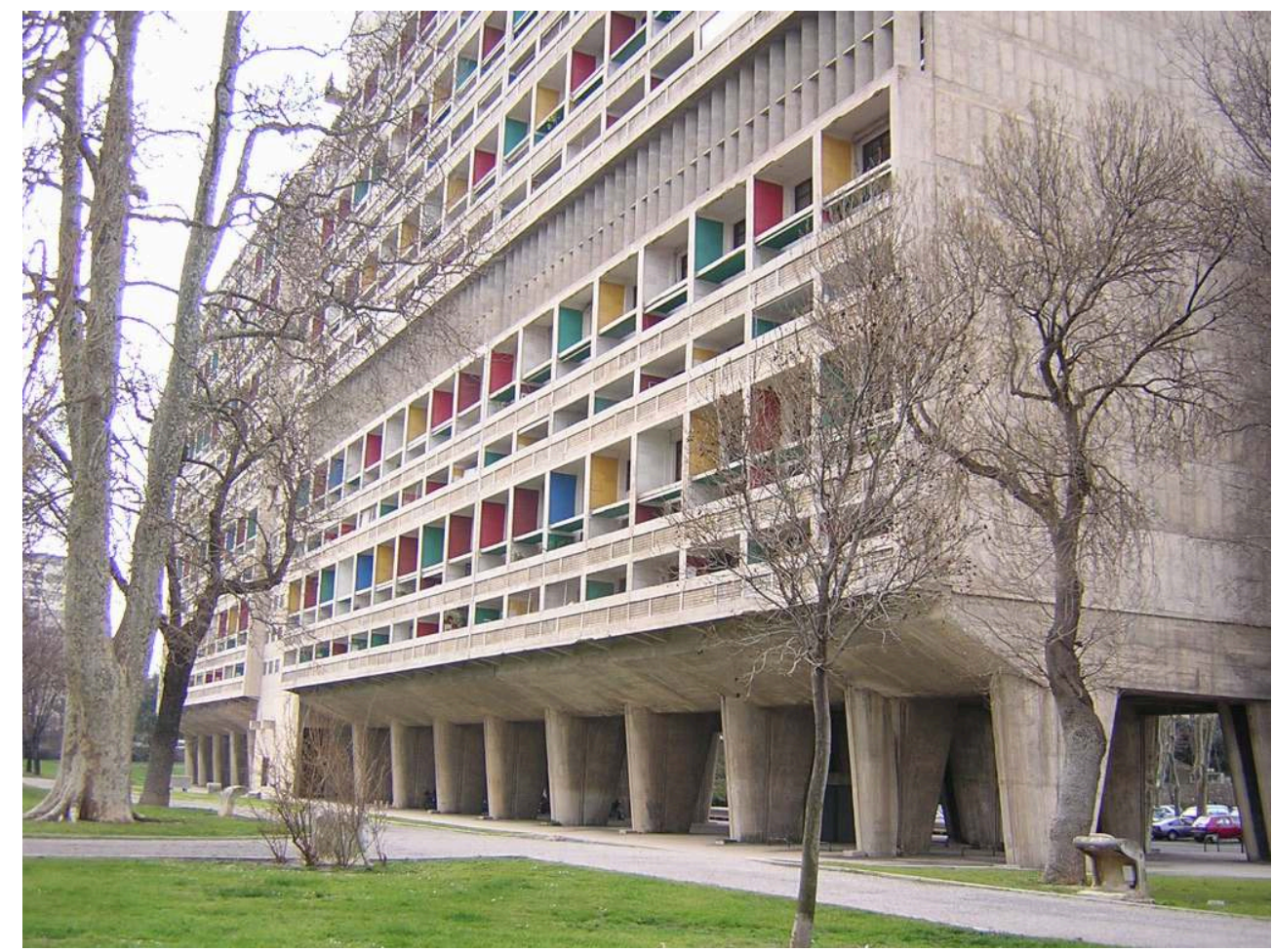

Les piliers de la cité radieuse

\section{BIBLIOGRAPHIE}

Andreotti Libero (dir.), Spielraum : W. Benjamin et l'architecture, Paris, Éd. de la Villette, 2011.

Aragon Louis, Le paysan de Paris, Paris, Éd. de la Nouvelle Revue française, 1926.

Arendt Hannah, Le système totalitaire (vol. 3 de Les Origines du totalitarisme), Jean-Loup Bourget, Robert Davreu et Patrick Lévy (trad.), Paris, Seuil, 1972.

Arendt Hannah, Condition de l'homme moderne, Georges Fradier (trad.), Paris, Calmann-Lévy (Agora), 1983.

Benjamin Walter, Enfance berlinoise, Jean Lacoste (trad.), Paris, Les Lettres Nouvelles / Maurice Nadeau, 1988.

Benjamin Walter, Paris, capitale du XIX ${ }^{e}$ siècle, Jean Lacoste (trad.), Paris, Éd. du Cerf, 1989.

Benjamin Walter, « Voyage en Italie » [1912], dans Écrits autobiographiques, Christophe Jouanlanne, Jean-François Poirier (trad.), Paris, Christian Bourgois, 1994.

Benjamin Walter, « Habiter sans laisser de traces », dans Images de pensée, Paris, Christian Bourgois, 1998. 
Benjamin Walter, « Journal de Moscou » [1927], dans Images de pensée, Jean-François Poirier, Jean Lacoste (trad.), Paris, Christian Bourgois, 1998.

Benjamin Walter, Lacis Asja, « Journal de Naples » [1925], dans Images de pensée, Paris, Christian Bourgois, 1998.

Benjamin Walter, « Le Surréalisme. Le dernier instantané de l'intelligentsia européenne », Maurice de Gandillac (trad.), revue par Pierre Rusch, dans Euvres II, Paris, Gallimard, 2000.

Benjamin Walter, Fragments : philosophiques, politiques, critiques, littéraires, Christophe Jouanlanne (trad.), Paris, PUF, 2001.

Benjamin Walter, Haschich à Marseille, Christophe Jouanlanne, Jean-François Poirier (trad.), Paris, Fata Morgana, 2013.

Berdet Marc, Fantasmagories du capital : L'invention de la ville-marchandise, Paris, Zones, 2013.

Déotte Jean-Louis, L'Homme de verre : esthétiques benjaminiennes, Paris/Montréal, L'Harmattan, 1988.

Déotte Jean-Louis, L'époque des appareils, Paris, Lignes \& Manifeste, 2004.

Déotte Jean-Louis, Walter Benjamin et la forme plastique, Paris, L'Harmattan, 2012.

Giedion Siegfried, Construire en France, construire en fer, construire en béton [1928], Paris, Éd. de la Villette, 2000.

Harvey David, Paris, capitale de la modernité, Matthieu Giroud (trad.), Paris, Les Prairies Ordinaires (Singulières modernités), 2012.

Monnier Gérard, Le Corbusier. Les Unités d'habitation en France, Paris, Belin (Les destinées du patrimoine), 2002.

Nancy Jean-Luc, La ville au loin, Strasbourg, Éd. la Phocide, 2011.

Proust Françoise, L'Histoire à contretemps : le temps historique chez Walter Benjamin, Paris, Librairie générale française, 1994.

Sbriglio Jacques, Le Corbusier. Habiter : de la villa Savoye à l'Unité d'habitation de Marseille [+ DVD], Paris, Actes Sud, 2009.

Teyssot Georges, « Responsive Envelopes: the Fabric of Climatic Islands », Appareil, $\mathrm{n}^{\circ} 11$, juin 2013, http://revues.mshparisnord.org/appareil/index.php/lodel/docannexe/image/1036/ index.php?id=1620.

\section{NOTES}

1. Libero Andreotti (dir.), Spielraum : W. Benjamin et l'architecture, Paris, Éd. de la Villette, 2011.

2. Hannah Arendt, Condition de l'homme moderne, Georges Fradier (trad.), Paris, Calmann-Lévy (Agora), 1983 ; Jean-Luc Nancy, La ville au loin, Strasbourg, Éd. La Phocide, 2011.

3. Jean-Louis Déotte, L'Homme de verre: esthétiques benjaminiennes, Paris/Montréal, L'Harmattan, 1988.

4. Françoise Proust, L'Histoire à contretemps : le temps historique chez Walter Benjamin, Paris, Librairie générale française, 1994.

5. Jean-Louis Déotte, Walter Benjamin et la forme plastique, Paris, L'Harmattan, 2012.

6. Siegfried Giedion, Construire en France, construire en fer, construire en béton [1928], Paris, Éd. de la Villette, 2000. 
7. Topologie: définition et explications : http://www.techno-science.net/? onglet=glossaire\&definition $=5151$

8. Georges Teyssot, «Responsive Envelopes: the Fabric of Climatic Island», Appareil, $\mathrm{n}^{\circ} 11$, juin 2013.

9. Walter Benjamin, "Le Surréalisme. Le dernier instantané de l'intelligentsia européenne ", Maurice de Gandillac (trad.), revue par Pierre Rusch, dans Euvres II, Paris, Gallimard, 2000, p. 134. Faut-il rappeler qu'à l'époque où il écrivait sur le surréalisme, Paris aurait abrité, d'après l'historien Graham Robb (Une histoire de Paris par ceux qui l'ont fait, 2010-2012), dix mille alchimistes !

10. Walter Benjamin, Fragments: philosophiques, politiques, critiques, littéraires, Christophe Jouanlanne (trad.), Paris, PUF, 2001, que je commente dans L'époque des appareils, Paris, Lignes \& Manifeste, 2004.

11. Walter Benjamin, Haschich à Marseille, Christophe Jouanlanne, Jean-François Poirier (trad.), Paris, Fata Morgana, 2013.

12. Walter Benjamin, Enfance berlinoise, Jean Lacoste (trad.), Paris, Les Lettres Nouvelles / Maurice Nadeau, 1988, en part. p. 110-111.

13. Walter Benjamin, «Voyage en Italie» [1912], dans Écrits autobiographiques, Christophe Jouanlanne, Jean-François Poirier (trad.), Paris, Christian Bourgois, 1994.

14. Sur ce point, voir également mon article: «W. Benjamin: la perspective comme fantasmagorie ", Varia, revue Appareil, février, 2014.

15. Hannah Arendt, Le système totalitaire (vol. 3 de Les Origines du totalitarisme), Jean-Louis Bourget, Robert Davreu et Patrick Lévy (trad.), Paris, Seuil, 1972.

16. Ce qui n'empêche pas l'utilisation réflexive, par certains groupes, du cinéma, à des fins politiques. Voir L'époque des appareils. Soit la politique de l'esthétique au sens de Benjamin.

17. Louis Aragon, Le Paysan de Paris, Paris, Éd. de la Nouvelle Revue française, 1926.

18. Ibid., p. 30-31; p. 32 ; p. 52-53; p. 67 ; p. 119.

19. Marc Berdet, Fantasmagories du capital : l'invention de la ville-marchandise, Paris, Zones, 2013. Et pour une critique d'Haussmann par un marxiste anglo-saxon contemporain : David Harvey, Paris, capitale de la modernité, Matthieu Giroud (trad.), Paris, Les Prairies Ordinaires (Singulières modernités), 2012. On peut noter, qu'en bon marxiste, Harvey cite Benjamin pour en limiter immédiatement la portée.

20. Walter Benjamin, Paris, capitale du XIX ${ }^{e}$ siècle, Jean Lacoste (trad.), Paris, Éditions du Cerf, 1989, p. 239, \& « Habiter sans laisser de traces », dans Images de pensée, p. 231.

21. Walter Benjamin, « Journal de Moscou » (1927), dans Images de pensée, Jean-François Poirier et Jean Lacoste (trad.), Paris, Christian Bourgois, 1998.

22. Walter Benjamin, « Journal de Naples » (1925), dans Images de pensée, op. cit.

23. Walter Benjamin, Asja Lacis, « Journal de Naples» (1925), dans Images de pensée.

24. Jacques Sbriglio, Le Corbusier. Habiter : de la villa Savoye à l'Unité d'habitation de Marseille, + DVD, Paris, Actes Sud, 2009, \& Gérard Monnier, Le Corbusier. Les Unités d'habitation en France, Paris, Belin (Les destinées du patrimoine), 2002. Les différentes luttes menées par les habitants sont relatées. 


\section{RÉSUMÉS}

Qu'est-ce que la politique pour Benjamin? Une politique de la ville. Quels sont ses idéaux-types (status) ? Entre le Paris des passages et Moscou la révolutionnaire? En quelle manière Benjamin pouvait-il annoncer les Unités d'habitation de Le Corbusier? Quels sont ses concepts pour comprendre l'articulation : habiter/espace public? Qu'est-ce qu'habiter pour lui ?

\section{INDEX}

Mots-clés : politique de l'espace, urbanisme, habitation, Paris, passage urbain Personnes citées : Le Corbusier

\section{AUTEUR}

\section{JEAN-LOUIS DÉOTTE}

Philosophe français, professeur à l'université Paris 8 